\title{
Stability Analysis of Hepatitis B Virus Model with Incomplete Immunization of HepB Vaccine
}

\author{
Yan Cheng, ${ }^{1,2}$ Qiuhui Pan, ${ }^{1,3}$ and Mingfeng $\mathrm{He}^{1}$ \\ ${ }^{1}$ School of Mathematical Sciences, Dalian University of Technology, Dalian 116024, China \\ ${ }^{2}$ Department of Mathematics, Tonghua Normal University, Tonghua 136000, China \\ ${ }^{3}$ School of Innovation Experiment, Dalian University of Technology, Dalian 116024, China
}

Correspondence should be addressed to Mingfeng He; mfhe@dlut.edu.cn

Received 25 March 2014; Accepted 19 May 2014; Published 12 June 2014

Academic Editor: Abdul Latif

Copyright (C) 2014 Yan Cheng et al. This is an open access article distributed under the Creative Commons Attribution License, which permits unrestricted use, distribution, and reproduction in any medium, provided the original work is properly cited.

\begin{abstract}
In this paper a HBV infection model with impulsive vaccination is considered. By using fixed point theorem and stroboscopic map we prove the existence of disease-free T-periodic solution. Also by comparative theorem of impulsive differential equation we get the global asymptotic stability of the disease-free periodic solution and permanence of the disease. Numerical simulations show the influence of parameters on the dynamics of HBV, which provided references for seeking optimal measures to control the transmission of HBV.
\end{abstract}

\section{Introduction}

Hepatitis B is a potentially life-threatening liver infection caused by the hepatitis B virus (HBV) and is a major global health problem. According to the data of World Health Organization (WHO), more than 2,000 million people have been infected with HBV and about 350 million remain infected chronically. Every year there are over 4 million acute clinical cases of HBV and about $25 \%$ of carriers. Hepatitis B causes about 1 million people to die from chronic active hepatitis, cirrhosis, or primary liver cancer annually [1].

The transmission of HBV occurs normally on contact with infected blood or body fluids. In high prevalence populations, transmission is largely vertical, that is, through mother to child during delivery or horizontal through household contact as skin breaches, open sores, or scratches in the early years of life. In contrast, HBV transmission in low endemicity populations typically occurs in adults via parenteral exposures and intravenous drug use or through sexual contact [2]. It can cause acute and chronic infection status. In acute infection status the individuals have highly infectiousness, and about $90 \%$ of adults who are infected with HBV will recover and be completely rid of the virus within six months. The rest of acute infectors turn into chronic infectors and acute hepatitis B occurs rarely in infants. The major routes of chronic hepatitis B infection are motherinfant vertical transmission and early childhood horizontal transmission [3]. Only a small fraction of chronic infectors could be cured completely [4]. Continuous chronic HBV infection exhibits various kinds of clinical symptoms, such as hepatocirrhosis and even hepatocellular carcinoma [5].

Vaccination is an effective control measures for HBV infection, an universal vaccination programme promoted in more than 170 countries since 1982 [6]. In New Zealand, the widespread introduction of infant hepatitis $B$ vaccination in 1988 led to a dramatic decline in cases of acute HBV infection [7]. From 2002, the Ministry of Health of China integrated the infant $\mathrm{HepB}$ vaccination into the national immunization program with vaccine provided entirely by the government. According to 2006-2010 hepatitis B control program, China will consistently strengthen the general newborn hepatitis $B$ vaccination and especially supply the first dose of 3-dose HepB series as soon as possible after birth [8].

Mathematical models have been used frequently to study the transmission dynamics of $\mathrm{HBV}$, and qualitative results on such models can be found. Anderson and May first used a simple mathematical model to illustrate the effects of carriers on the transmission of HBV [9]. Thornley et al. 
[10] develop a hepatitis B mathematical model, proposed by Medley et al. [11], that was used to develop a strategy for eliminating the HBV spread in New Zealand in 2008. Zou et al. also proposed a mathematical model to understand the transmission dynamics and prevalence of $\mathrm{HBV}$ in mainland China [12]. Pang et al. [13] develop a model to explore the impact of vaccination and other controlling measures of HBV infection and a mathematical model which describes the spread of HBV is formulated in [14]. Zou et al. [15] promote an age structure model to predict the dynamics of HBV transmission and evaluate the long-term effectiveness of the vaccination programme in China. Wang et al. proposed and analyzed the hepatitis $B$ virus infection in a diffusion model confined to a finite domain [16]. A hepatitis B virus (HBV) model with spatial diffusion and saturation response of the infection rate is investigated by $\mathrm{Xu}$ and $\mathrm{Ma}$ [17]. Transmission model of hepatitis $B$ virus with the migration effect is presented by Khan et al. [18] who analyzed the effect of immigrants in the model to study the effect of immigrants for the host population.

In the above literatures, most models involving НерB vaccine strategy often assumed that the vaccine is completely effective in preventing the infection of vaccinated individuals. In fact, it is well known to all that the HepB vaccine should be taken in three doses at 0,1 , and 6 months. Usually $30-$ $50 \%$ of individuals will gain anti-HBs antibody after the first dose, $80-90 \%$ will gain after the second dose, and almost all the individuals will have high anti-HBs concentrations one month after the last dose that $99.8 \%$ of vaccinees gained antiHBs antibody [19]. So, as soon as the susceptible individuals begin the vaccination process, they are different from susceptible individuals. But they should also be distinguished from recovered individuals who have immunity against the disease. It means that a few of vaccinated individuals may still be susceptible to infection, but they will be infected at a lower rate than unvaccinated susceptible individuals. Epidemic models including incomplete immune compartment $V$ have been studied in [20-24], but the HBV transmission with the incomplete HepB vaccine immune is rarely considered.

Motivated by the above consideration, and based on the natural course of HBV infection, we promote a novel model to describe the transition dynamic of HBV. It is more reasonable to consider the impulsive vaccination strategy for the susceptible individuals; there are fewer literatures that researched HBV infection with impulsive vaccination already $[25,26]$. We also consider the incomplete immune compartment in our model.

The remaining parts of this paper are organized as follows. In Section 2, we formulate the model. In Section 3, we study the global asymptotic stability of disease-free periodic solution and the conditions for the permanence of the disease by comparison techniques. Numerical simulations are presented in Section 4. In Section 5, we conclude this paper with some remarks.

\section{Modeling}

Based on the fact that HepB vaccination is not completely effective, we improve the model of Zou et al. [12] in three aspects. Firstly, we considered that vaccinated individuals may still be susceptible to infection. Secondly, we studied impulsive vaccination strategy for the susceptible individuals. Finally, we add a latent period $\tau$. We divide the population into six epidemiological groups: the susceptible individuals to infection $S$; latently infected $L$; those acute infectors $I_{1}$; chronic sufferers $I_{2}$; and recovered $R ; V$ denotes the density of vaccinees who have begun the vaccination process. The individuals in $V$ are different from those in $S$ and $R$; the immune system will create antibody of HBV because vaccination doses are taken during this process, but it may not be in a fully protective level. According to the characteristics of HBV transmission, the flow diagram is shown in Figure 1.

The mathematical model of the transmission dynamics and prevalence of $\mathrm{HBV}$ is as follows:

$$
\begin{aligned}
& S^{\prime}(t)= \mu \omega\left(1-v I_{2}\right)+\varphi V-\left(\mu+\beta I_{1}+\varepsilon \beta I_{2}\right) S, \\
& L^{\prime}(t)=\left(\beta I_{1}+\varepsilon \beta I_{2}\right)(S+\theta V)-e^{-\mu \tau} \\
& \times\left(\beta I_{1}(t-\tau)+\varepsilon \beta I_{2}(t-\tau)\right) \\
& \times(S(t-\tau)+\theta V(t-\tau))-\mu L, \\
& I_{1}^{\prime}(t)= e^{-\mu \tau}\left(\beta I_{1}(t-\tau)+\varepsilon \beta I_{2}(t-\tau)\right) \\
& \times(S(t-\tau)+\theta V(t-\tau))-\left(\mu+\gamma_{1}\right) I_{1}, \\
& I_{2}^{\prime}(t)= \mu \omega v I_{2}+q \gamma_{1} I_{1}-\left(\mu+\mu_{1}+\gamma_{2}\right) I_{2}, \\
& V^{\prime}(t)= \mu(1-\omega)-\theta\left(\beta I_{1}+\varepsilon \beta I_{2}\right) V-(\mu+\varphi) V, \\
& R^{\prime}(t)=(1-q) \gamma_{1} I_{1}+\gamma_{2} I_{2}-\mu R, \\
& t \neq n T, \quad n \in Z^{+} \\
& S\left(t^{+}\right)=(1-p) S(t), \\
& V\left(t^{+}\right)=V(t)+p S(t), \\
& t=n T, \quad n \in Z^{+} .
\end{aligned}
$$

In these equations, all the parameters are nonnegative. $\mu$ is the per capita natural death rate and the birth rate of newborns, and $\mu_{1}$ is the per capita disease-induced death rate. $\beta$ is the transmission coefficient of acute $\mathrm{HBV}$, and $\varepsilon \beta$ is the reduced transmission coefficient of chronic HBV. $\omega$ is the proportion of births without successful vaccination. $\gamma_{1}$ is the rate at which individuals leave the acute infection class; a proportion $q$ of acute infection individuals become chronic carriers and other proportion (1-q) rid of $\mathrm{HBV}$, move directly to immunity class R. $\gamma_{2}$ is the recovery conversion rate of chronic infectors. $v$ is the proportion of unimmunized children born to chronic carrier mothers who have been infected (ignore perinatal infection of children born to mothers with acute infection). That $\mu \omega v I_{2}$ denotes newborns who have been infected in perinatal infection and in chronic infection compartment, the rest $\mu \omega\left(1-v I_{2}\right)$ newborns become susceptible individuals. 


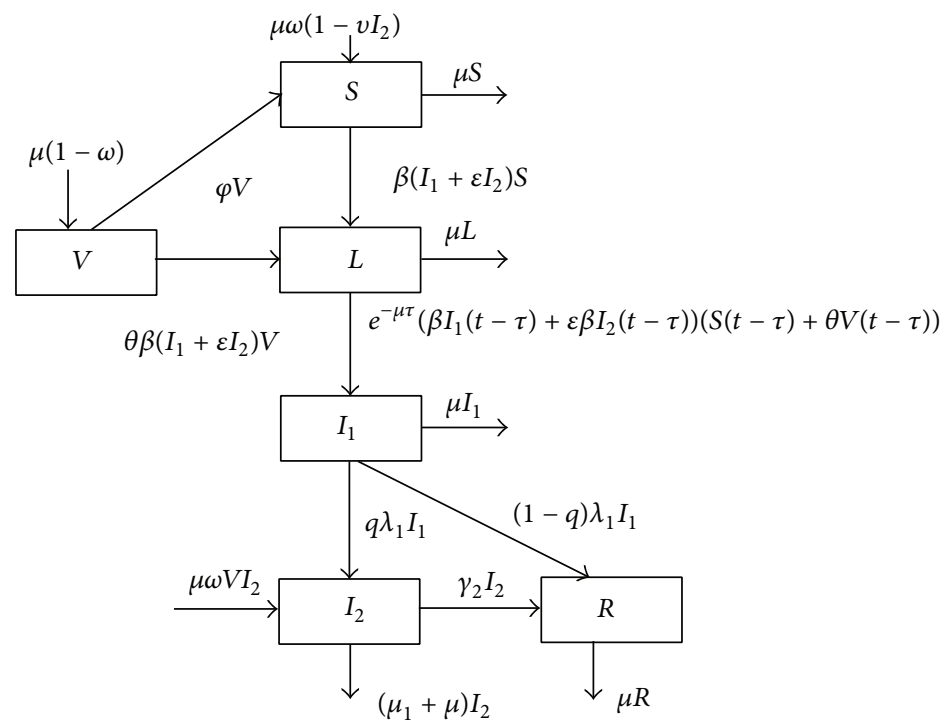

FIgURE 1: Flow diagram of HBV transmission in a population.

The factor $\theta(0<\theta<1)$ reflects the efficacy of vaccine, which means that the vaccine is not completely effective and that the vaccinated individuals have only partial immunity. For the vaccinated individuals, let $\varphi$ denote the per capita rate coefficient at which the immunity wears off, which implies that the vaccinated individuals have the temporary immunity. $p(0<p<1)$ is the fraction of impulsive vaccination, and $\tau$ is latent period. The period of vaccination is $T$, and the vaccination is dose at time $t=n T, n \in N=\{1,2,3, \ldots\}$.

Since the equations for the variables $L$ and $R$ in model (1) are both independent of other equations, then the dynamical behavior of (1) is determined by the following system:

$$
\begin{aligned}
& S^{\prime}(t)= \mu \omega\left(1-v I_{2}\right)+\varphi V-\left(\mu+\beta I_{1}+\varepsilon \beta I_{2}\right) S, \\
& I_{1}^{\prime}(t)= e^{-\mu \tau}\left(\beta I_{1}(t-\tau)+\varepsilon \beta I_{2}(t-\tau)\right) \\
& \times(S(t-\tau)+\theta V(t-\tau))-\left(\mu+\gamma_{1}\right) I_{1}, \\
& I_{2}^{\prime}(t)= \mu \omega v I_{2}+q \gamma_{1} I_{1}-\left(\mu+\mu_{1}+\gamma_{2}\right) I_{2}, \\
& V^{\prime}(t)= \mu(1-\omega)-\theta\left(\beta I_{1}+\varepsilon \beta I_{2}\right) V-(\mu+\varphi) V, \\
& t \neq n T, \quad n \in Z^{+}
\end{aligned}
$$

$$
\begin{aligned}
S\left(t^{+}\right) & =(1-p) S(t), \\
V\left(t^{+}\right) & =V(t)+p S(t), \\
t & =n T, \quad n \in Z^{+} .
\end{aligned}
$$

The initial conditions for system (2) take the forms

$$
\begin{gathered}
S\left(0^{+}\right)=S^{0}, \quad I_{1}\left(0^{+}\right)=I_{1}^{0}, \\
I_{2}\left(0^{+}\right)=I_{2}^{0}, \quad V\left(0^{+}\right)=V^{0}, \\
\Phi=\left(S(\theta), I_{1}(\theta), I_{2}(\theta), V(\theta) \in C\left([-\tau, 0], R_{+}^{4}\right)\right) .
\end{gathered}
$$

For $\left(S+I_{1}+I_{2}+V\right)^{\prime} \leq \mu\left[1-\left(S+I_{1}+I_{2}+V\right)\right]$ it follows that $\sup _{t \rightarrow \infty}\left(S+I_{1}+I_{2}+V\right) \leq 1$. Set $\Omega=\left\{\left(S+I_{1}+I_{2}+V\right) \in R_{+}^{4}\right.$ : $\left.S+I_{1}+I_{2}+V \leq 1\right\}$ is positively invariant of system (2).

Subsequently, we introduce the following lemma, which is useful for the later proof.

Lemma 1. Consider the following impulsive differential equation:

$$
\begin{gathered}
S^{\prime}(t)=\mu \omega-\mu S+\varphi V, \\
V^{\prime}(t)=\mu(1-\omega)-(\mu+\varphi) V \\
t \neq n T, \quad n \in Z^{+} \\
S\left(t^{+}\right)=(1-p) S(t), \\
V\left(t^{+}\right)=V(t)+p S(t), \\
t=n T, \quad n \in Z^{+} .
\end{gathered}
$$

Then the above system has a unique positive periodic solution given by

$$
\begin{aligned}
& \widetilde{S}(t)=\frac{\mu \omega+\varphi}{\mu+\varphi}-\left(V^{*}-\frac{\mu(1-\omega)}{\mu+\varphi}\right) e^{-(\mu+\varphi)(t-n T)}, \\
& \widetilde{V}(t)=\frac{\mu(1-\omega)}{\mu+\varphi}+\left(V^{*}-\frac{\mu(1-\omega)}{\mu+\varphi}\right) e^{-(\mu+\varphi)(t-n T)} .
\end{aligned}
$$

Consider that $n T<t \leq(n+1) T$, which is globally asymptotically stable, where

$$
\begin{aligned}
S^{*} & =\frac{(1-p)(\varphi+\mu \omega)\left(1-e^{(\mu+\varphi) T}\right)}{(\mu+\varphi)\left[1-(1-p) e^{-(\mu+\varphi) T}\right]}, \\
V^{*} & =\frac{p(\mu+\varphi)+\mu(1-p)(1-\omega)\left[1-e^{-(\mu+\varphi) T}\right]}{(\mu+\varphi)\left[1-(1-p) e^{-(\mu+\varphi) T}\right]} .
\end{aligned}
$$


Proof. It is easy to obtain the analytical solution of (4) between pulses as follows:

$$
\begin{aligned}
& S(t)= \frac{\varphi+\omega \mu}{\mu+\varphi}+\left[S\left(n T^{+}\right)+V\left(n T^{+}\right)-1\right] e^{-\mu(t-n T)} \\
&-\left(V\left(n T^{+}\right)-\frac{\mu(1-\omega)}{\mu+\varphi}\right) e^{-(\mu+\varphi)(t-n T),} \\
& V(t)= \frac{\mu(1-\omega)}{\mu+\varphi}+\left(V\left(n T^{+}\right)-\frac{\mu(1-\omega)}{\mu+\varphi}\right) e^{-(\mu+\varphi)(t-n T)}, \\
& n T \leq t \leq(n+1) T .
\end{aligned}
$$

Furthermore, after each successive pulse, we can deduce the following stroboscopic map:

$$
\begin{aligned}
S[ & \left.(n+1) T^{+}\right] \\
= & (1-p)\left\{\frac{\varphi+\omega \mu}{\mu+\varphi}+\left[S\left(n T^{+}\right)+V\left(n T^{+}\right)-1\right] e^{-\mu T}\right\} \\
& -(1-p)\left(V\left(n T^{+}\right)-\frac{\mu(1-\omega)}{\mu+\varphi}\right) e^{-(\mu+\varphi) T}, \\
v\left[(n+1) T^{+}\right] & \\
= & \frac{\mu(1-\omega)}{\mu+\varphi}+p S\left(n T^{+}\right) e^{-\mu T}+\frac{p(\varphi+\omega \mu)}{\mu+\varphi}\left(1-e^{-\mu T}\right) \\
& +\left(V\left(n T^{+}\right)-\frac{\mu(1-\omega)}{\mu+\varphi}\right)\left[(1-p) e^{-(\mu+\varphi) T}+p e^{-\mu T}\right] .
\end{aligned}
$$

Denote $x_{n}=S\left(n T^{+}\right), y_{n}=V\left(n T^{+}\right)$; then we have the following equations:

$$
\begin{aligned}
x_{(n+1)}= & (1-p)\left\{\frac{\varphi+\omega \mu}{\mu+\varphi}+\left[x_{n}+y_{n}-1\right] e^{-\mu T}\right\} \\
& -(1-p)\left(y_{n}-\frac{\mu(1-\omega)}{\mu+\varphi}\right) e^{-(\mu+\varphi) T}, \\
y_{(n+1)}= & \frac{\mu(1-\omega)}{\mu+\varphi}+p x_{n} e^{-\mu T}+\frac{p(\varphi+\omega \mu)}{\mu+\varphi}\left(1-e^{-\mu T}\right) \\
& +\left(y_{n}-\frac{\mu(1-\omega)}{\mu+\varphi}\right)\left[(1-p) e^{-(\mu+\varphi) T}+p e^{-\mu T}\right] .
\end{aligned}
$$

The unique positive fixed point is $\left(x^{*}, y^{*}\right)$, where

$$
\begin{aligned}
x^{*} & =\frac{(1-p)(\varphi+\mu \omega)\left(1-e^{(\mu+\varphi) T}\right)}{(\mu+\varphi)\left[1-(1-p) e^{-(\mu+\varphi) T}\right]}, \\
y^{*} & =\frac{p(\mu+\varphi)+\mu(1-p)(1-\omega)\left[1-e^{-(\mu+\varphi) T}\right]}{(\mu+\varphi)\left[1-(1-p) e^{-(\mu+\varphi) T}\right]} .
\end{aligned}
$$

So, (4) has a unique period solution with initial values of $S\left(0^{+}\right)=x^{*}, V\left(0^{+}\right)=y^{*}$.
In the following we prove the global stability of the period solution, and it suffices to prove the global stability of the fixed point $\left(x^{*}, y^{*}\right)$. The proof is similar to the proof of Lemma 2.1 in [22], so we omit the subsequent proof.

Lemma 2 (see [27]). Assume that the sequence $\left\{t_{k}\right\}$ satisfies $0 \leq t_{0}<t_{1}<t_{2} \cdots$ with $\lim _{\mathrm{k} \rightarrow \mathrm{t}_{\mathrm{k}}}=\infty$. Let $f(t, x): R_{+} \rightarrow R^{n}$ be quasi-monotone nondecreasing in $x$ for each $t$, and $\psi_{k}(u) \in$ $C\left[R^{n}, R^{n}\right]$ is nondecreasing in $u$ for $k=1,2, \ldots$. Suppose that $u(t), v(t) \in P C\left(\left[t_{0}, \infty\right], R^{n}\right)$ satisfy

$$
\begin{array}{ll}
D^{+} u(t) \leq f(t, u(t)), & t \geq t_{0}, \\
u\left(t_{k}^{+}\right) \leq \psi_{k}\left(u\left(t_{k}\right)\right), & k \in N, \\
D^{+} v(t) \geq f(t, v(t)), & t \geq t_{0}, \\
v\left(t_{k}^{+}\right) \geq \psi_{k}\left(v\left(t_{k}\right)\right), \quad k \in N .
\end{array}
$$

Then $u_{0} \leq v_{0}$ implies that $u(t) \leq v(t)$ for $t \geq t_{0}$.

Lemma 3 (see [28]). Consider the following equation: $u^{\prime}(t)=$ $a_{1} u(t-\tau)-a_{2} u(t)$, where $a_{1}>0, a_{2}>0, \tau>0$, and $u(t)>0$ for $-\tau \leq t \leq 0$. We have

$$
\lim _{t \rightarrow \infty} u(t)= \begin{cases}0, & \text { if } a_{1}<a_{2}, \\ +\infty, & \text { if } a_{1}>a_{2} .\end{cases}
$$

\section{Stability and Persistence}

3.1. Global Stability of the Disease-Free Periodic Solution. Now we will prove the disease-free periodic solution $(\widetilde{S}(t)$, $0,0, \widetilde{V}(t))$ is locally stable and globally attractive. We first demonstrate the existence of the disease-free periodic solution, in which infectious individuals are entirely absent from the population permanently; that is, $I_{1}(t) \equiv 0$ and $I_{2}(t) \equiv 0$ for all $t>0$. Under this condition, the growth of susceptible individuals must satisfy

$$
\begin{aligned}
S^{\prime}(t)= & \mu \omega-\mu S+\varphi V, \\
V^{\prime}(t)= & \mu(1-\omega)-(\mu+\varphi) V, \\
t & \neq n T, \quad n \in Z^{+} \\
S\left(t^{+}\right) & =(1-p) S(t), \\
V\left(t^{+}\right) & =V(t)+p S(t), \\
t & =n T, \quad n \in Z^{+} .
\end{aligned}
$$

By Lemma 1, we obtain the periodic solution of system (13):

$$
\begin{aligned}
& \widetilde{S}(t)=\frac{\mu \omega+\varphi}{\mu+\varphi}-\left(V^{*}-\frac{\mu(1-\omega)}{\mu+\varphi}\right) e^{-(\mu+\varphi)(t-n T)}, \\
& \widetilde{V}(t)=\frac{\mu(1-\omega)}{\mu+\varphi}+\left(V^{*}-\frac{\mu(1-\omega)}{\mu+\varphi}\right) e^{-(\mu+\varphi)(t-n T)}
\end{aligned}
$$


where

$$
\begin{aligned}
S^{*} & =\frac{(1-p)(\varphi+\mu \omega)\left(1-e^{(\mu+\varphi) T}\right)}{(\mu+\varphi)\left[1-(1-p) e^{-(\mu+\varphi) T}\right]} \\
V^{*} & =\frac{p(\mu+\varphi)+\mu(1-p)(1-\omega)\left[1-e^{-(\mu+\varphi) T}\right]}{(\mu+\varphi)\left[1-(1-p) e^{-(\mu+\varphi) T}\right]}
\end{aligned}
$$

is globally asymptotically stable. Hence, the system (2) has a disease-free periodic solution $(\widetilde{S}(t), 0,0, \widetilde{V}(t))$.

Denote

$$
\begin{aligned}
& \mathfrak{R}_{1}=\frac{e^{-\mu \tau} \int_{0}^{T} \beta(\widetilde{S}+\theta \widetilde{V}) d t}{T\left[\mu+(1-q) \gamma_{1}\right]}, \\
& \mathfrak{R}_{2}=\frac{e^{-\mu \tau} \int_{0}^{T} \varepsilon \beta(\widetilde{S}+\theta \widetilde{V}) d t}{T\left[(1-\omega v) \mu+\mu_{1}+\gamma_{2}\right]} .
\end{aligned}
$$

Theorem 4. Let $\left(S(t), I_{1}(t), I_{2}(t), V(t)\right)$ be any solution of (2); then the disease-free periodic solution $(\widetilde{S}(t), 0,0, \widetilde{V}(t))$ is globally asymptotically stable provided that $\mathfrak{R}_{1}<1$ and $\mathfrak{R}_{2}<$ 1 .

Proof. Since $\mathfrak{R}_{1}<1$ and $\mathfrak{R}_{2}<1$, we can choose $\varepsilon_{1}>0$ sufficiently small such that

$$
\begin{aligned}
& e^{-\mu \tau} \int_{0}^{T}\left[\beta(\widetilde{S}+\theta \widetilde{V})+\beta \varepsilon_{1}(1+\theta)\right] d t \\
& <T\left[\mu+(1-q) \gamma_{1}\right], \\
& e^{-\mu \tau} \int_{0}^{T}\left[\varepsilon \beta(\widetilde{S}+\theta \widetilde{V})+\varepsilon \beta \varepsilon_{1}(1+\theta)\right] d t \\
& <T\left[(1-\omega v) \mu+\mu_{1}+\gamma_{2}\right] .
\end{aligned}
$$

From the equations of system (2), we have $S^{\prime}(t)<\mu \omega-\mu S+\varphi V$ and $V^{\prime}(t)<\mu(1-\omega)-(\mu+\varphi) V$; then we consider the following comparison system with pulse:

$$
\begin{aligned}
s^{\prime}(t) & =\mu \omega-\mu s+\varphi v, \\
v^{\prime}(t) & =\mu(1-\omega)-(\mu+\varphi) v, \\
& \neq n T, \quad n \in Z^{+} \\
s\left(t^{+}\right) & =(1-p) s(t), \\
v\left(t^{+}\right) & =v(t)+p s(t), \\
t & =n T, \quad n \in Z^{+} .
\end{aligned}
$$

In view of Lemma 1, we obtain

$$
\begin{aligned}
& \widehat{s}(t)=\frac{\mu \omega+\varphi}{\mu+\varphi}-\left(v^{*}-\frac{\mu(1-\omega)}{\mu+\varphi}\right) e^{-(\mu+\varphi)(t-n T)}, \\
& \widehat{v}(t)=\frac{\mu(1-\omega)}{\mu+\varphi}+\left(v^{*}-\frac{\mu(1-\omega)}{\mu+\varphi}\right) e^{-(\mu+\varphi)(t-n T)},
\end{aligned}
$$

where

$$
\begin{aligned}
s^{*} & =\frac{(1-p)(\varphi+\mu \omega)\left(1-e^{(\mu+\varphi) T}\right)}{(\mu+\varphi)\left[1-(1-p) e^{-(\mu+\varphi) T}\right]}, \\
v^{*} & =\frac{p(\mu+\varphi)+\mu(1-p)(1-\omega)\left[1-e^{-(\mu+\varphi) T}\right]}{(\mu+\varphi)\left[1-(1-p) e^{-(\mu+\varphi) T}\right]} .
\end{aligned}
$$

By Lemma 2 there exists an integer $k_{1}$, such that

$$
\begin{aligned}
& S(t) \leq s(t)<\widehat{s}(t)+\varepsilon_{1}=S_{\Delta}, \\
& V(t) \leq v(t)<\widehat{v}(t)+\varepsilon_{1}=V_{\Delta}, \\
& n T<t \leq(n+1) T, \quad n>k_{1} .
\end{aligned}
$$

Furthermore, from the second and third equations, we have

$$
\begin{aligned}
& I_{1}^{\prime}(t)+I_{2}^{\prime}(t) \\
& \leq\left[e^{-\mu \tau} \beta\left(S_{\Delta}+\theta V_{\Delta}\right) I_{1}(t-\tau)-\left(\mu+(1-q) \gamma_{1}\right) I_{1}(t)\right] \\
& +\left[\varepsilon \beta e^{-\mu \tau}\left(S_{\Delta}+\theta V_{\Delta}\right) I_{2}(t-\tau)\right. \\
& \left.\quad-\left(\mu-\mu v \omega+\mu_{1}+\gamma_{2}\right) I_{2}(t)\right]
\end{aligned}
$$

for $t \neq n T, n>k_{1}$. Then, according to (17) and Lemma 3, we have $I_{1}^{\prime}(t)+I_{2}^{\prime}(t) \leq 0$. So, $\lim _{k \rightarrow \infty}\left(I_{1}(t)+I_{2}(t)\right)=0$; there must exist an integer $k_{2}>k_{1}$, such that $I_{1}(t)<\varepsilon_{2}, I_{2}(t)<\varepsilon_{3}$ for all $t>k_{2} T$.

When $t>k_{2} T$, from the first equation of system (2), we have

$$
\begin{gathered}
S^{\prime}(t)>\mu \omega\left(1-v \varepsilon_{3}\right)+\varphi V-\left(\mu+\beta \varepsilon_{2}+\varepsilon \beta \varepsilon_{3}\right) S, \\
V^{\prime}(t)>\mu(1-\omega)-\theta\left(\beta \varepsilon_{2}+\varepsilon \beta \varepsilon_{3}\right) V-(\mu+\varphi) V .
\end{gathered}
$$

Consider the following comparison impulsive differential equation for all $t>k_{2} T$ :

$$
\begin{array}{r}
s_{1}\left(t^{+}\right)=(1-p) s_{1}(t), \\
v_{1}\left(t^{+}\right)=v_{1}(t)+p s_{1}(t), \\
t=n T, \quad n \in Z^{+} .
\end{array}
$$$$
s_{1}^{\prime}(t)=\mu \omega\left(1-v \varepsilon_{3}\right)+\varphi v_{1}-\left(\mu+\beta \varepsilon_{2}+\varepsilon \beta \varepsilon_{3}\right) s_{1}
$$$$
v_{1}^{\prime}(t)=\mu(1-\omega)-\theta\left(\beta \varepsilon_{2}+\varepsilon \beta \varepsilon_{3}\right) v_{1}-(\mu+\varphi) v_{1},
$$$$
t \neq n T, \quad n \in Z^{+}
$$ 
By Lemma 1, we have the unique periodic solution of system (25) given by

$$
\begin{aligned}
\widetilde{s}_{1}= & \frac{\mu \omega\left(1-v \varepsilon_{3}\right)+\varphi}{\varphi+\mu+\beta \varepsilon_{2}+\varepsilon \beta \varepsilon_{3}} \\
& -\left(v_{1}^{*}-\frac{\mu(1-\omega)}{(\mu+\varphi) \theta\left(\beta \varepsilon_{2}+\varepsilon \beta \varepsilon_{3}\right)}\right) \\
& \times e^{-\left[\theta\left(\beta \varepsilon_{2}+\varepsilon \beta \varepsilon_{3}\right)+\mu+\varphi\right](t-n T)}, \\
\widetilde{v}_{1}= & \frac{\mu(1-\omega)}{(\mu+\varphi) \theta\left(\beta \varepsilon_{2}+\varepsilon \beta \varepsilon_{3}\right)} \\
& +\left(v_{1}^{*}-\frac{\mu(1-\omega)}{(\mu+\varphi) \theta\left(\beta \varepsilon_{2}+\varepsilon \beta \varepsilon_{3}\right)}\right) \\
& \times e^{-\left[\theta\left(\beta \varepsilon_{2}+\varepsilon \beta \varepsilon_{3}\right)+\mu+\varphi\right](t-n T)},
\end{aligned}
$$

where

$$
\begin{gathered}
s_{1}^{*}=\frac{(1-p)\left(\mu \omega\left(1-v \varepsilon_{3}\right)+\varphi\right)\left(1-e^{\left(\theta\left(\beta \varepsilon_{2}+\varepsilon \beta \varepsilon_{3}\right)+\mu+\varphi\right) T}\right)}{\left(\varphi+\mu+\beta \varepsilon_{2}+\varepsilon \beta \varepsilon_{3}\right)\left[1-(1-p) e^{-\left(\theta\left(\beta \varepsilon_{2}+\varepsilon \beta \varepsilon_{3}\right)+\mu+\varphi\right) T}\right]}, \\
v_{1}^{*}=\left(p\left(\varphi+\mu+\beta \varepsilon_{2}+\varepsilon \beta \varepsilon_{3}\right)\right. \\
\left.+\mu(1-p)(1-\omega)\left[1-e^{-\left(\theta\left(\beta \varepsilon_{2}+\varepsilon \beta \varepsilon_{3}\right)+\mu+\varphi\right) T}\right]\right) \\
\times\left(\left(\varphi+\mu+\beta \varepsilon_{2}+\varepsilon \beta \varepsilon_{3}\right)\right. \\
\left.\times\left[1-(1-p) e^{-\left(\theta\left(\beta \varepsilon_{2}+\varepsilon \beta \varepsilon_{3}\right)+\mu+\varphi\right) T}\right]\right)^{-1} .
\end{gathered}
$$

By comparison theorem, there exists an integer $k_{3}>k_{2}$ such that

$$
\begin{array}{r}
S(t)>s_{1}(t)>\widetilde{s}_{1}-\varepsilon_{4}, \\
V(t)>v_{1}(t)>\widetilde{v}_{1}-\varepsilon_{4}, \\
n T<t \leq(n+1) T .
\end{array}
$$

Because $\varepsilon_{1}, \varepsilon_{2}, \varepsilon_{3}$, and $\varepsilon_{4}$ are sufficiently small, it follows from (22) and (28) that $\lim _{t \rightarrow \infty} S(t)=S^{*}(t), \lim _{t \rightarrow \infty} V(t)=V^{*}(t)$. Therefore, the disease-free solution $(\widetilde{S}(t), 0,0, \widetilde{V}(t))$ of system (2) is globally attractive. The proof is completed.

Theorem 4 determines the global attractiveness of (2) in $\Omega$ for the cases $\Re_{1}<1$ and $\Re_{2}<1$. Its epidemiological implication is that the infectious population vanishes in time so the disease dies out.

3.2. Persistence. In this section we say the disease is endemic if the infectious population persists above a certain positive level for sufficiently large time. The endemicity of the disease can be well captured and studied through the notion of uniform persistence.

Definition 5. System (2) is said to be uniformly persistent if there exist positive constants $M_{i} \geq m_{i}, i=1,2,3$ (both are independent of the initial values), such that every solution $\left(S(t), I_{1}(t), I_{2}(t), V(t)\right)$ with positive initial conditions of system (2) satisfies

$$
\begin{gathered}
m_{1} \leq S(t) \leq M_{1}, \\
m_{2} \leq I_{1}(t)+I_{2}(t) \leq M_{2}, \\
m_{3} \leq V(t) \leq M_{3} .
\end{gathered}
$$

Theorem 6. If $\Re_{1}>1$ and $\Re_{2}>1$, then there is a positive constant $m_{I}$ such that each positive solution $\left(I_{1}(t), I_{2}(t)\right)$ of system (2) satisfies $I_{1}(t)+I_{2} \geq m_{I}$ for all $t$ sufficiently large.

Proof. Let $\left(S(t), I_{1}(t), I_{2}(t), V(t)\right)$ be any solution with initial values of system (2); then it is obvious that $S(t) \leq 1, I_{1}(t) \leq 1$, $I_{2}(t) \leq 1$, and $V(t) \leq 1$ for all $t>0$. We are left to prove that there exist positive constants $m_{S}, m_{I}, m_{V}$, and $t_{0}\left(t_{0}\right.$ is sufficiently large) such that $S(t) \geq m_{S}, I_{1}(t)+I_{2} \geq m_{I}$, and $V(t) \geq m_{V}$ for all $t>t_{0}$.

Firstly, from the first equation of system (2), we have

$$
\begin{gathered}
S^{\prime}(t)>\mu \omega(1-v)-(\mu+\beta+\varepsilon \beta) S+\varphi V, \\
V^{\prime}(t)>\mu(1-\omega)-(\mu+\varphi+\theta(\beta+\varepsilon \beta)) V .
\end{gathered}
$$

Consider the following comparison equations:

$$
\begin{gathered}
u_{1}^{\prime}(t)=\mu \omega(1-v)-(\mu+\beta+\varepsilon \beta) S+\varphi V, \\
u_{2}^{\prime}(t)=\mu(1-\omega)-(\mu+\varphi+\theta(\beta+\varepsilon \beta)) V, \\
t \neq n T, \quad n \in Z^{+} \\
u_{1}\left(t^{+}\right)=(1-p) s(t), \\
u_{2}\left(t^{+}\right)=v(t)+p s(t), \\
t=n T, \quad n \in Z^{+} .
\end{gathered}
$$

By Lemma 1 and the comparison theorem there exist $u_{1}^{*}, u_{2}^{*}$; we know that for any sufficiently small $\varepsilon_{0}>0$, there exists a $t_{0}\left(t_{0}\right.$ is sufficiently large) such that

$$
\begin{gathered}
S(t) \geq u_{1}(t)>u_{1}^{*}(t)-\varepsilon_{0}=m_{S}>0, \\
V(t) \geq u_{2}(t)>u_{2}^{*}(t)-\varepsilon_{0}=m_{V}>0 .
\end{gathered}
$$

Next, we will prove that there exist $m_{I}>0$ and a sufficiently large $t_{0}$ such that $I_{1}(t)+I_{2}(t) \geq m_{I}$ for all $t>t_{0}$.

Since $\mathfrak{R}_{1}>1$ and $\mathfrak{R}_{2}>1$, there exist $m_{I}^{*}>0$ and $\bar{\varepsilon}>0$ sufficiently small such that

$$
\begin{gathered}
e^{-\mu \tau} \int_{0}^{T}\left[\beta\left(\eta_{1}+\theta \eta_{2}\right)\right]-T\left(\mu+(1-q) \gamma_{1}\right)>0, \\
e^{-\mu \tau} \int_{0}^{T}\left[\varepsilon \beta\left(\eta_{1}+\theta \eta_{2}\right)\right]+T\left[\mu v \omega-\left(\mu+\mu_{1}+\gamma_{2}\right)\right]>0,
\end{gathered}
$$

where $\eta_{1}$ and $\eta_{2}$ are as in (38).

We claim that for any $t_{0}>0$, it is impossible that $I_{1}(t)+$ $I_{2}(t)<m_{I}^{*}$ for all $t \geq t_{0}$. Suppose that the claim is not valid. 
There exists a $t_{0}>0$ such that $I_{1}(t)+I_{2}(t)<m_{I}^{*}$ for all $t \geq t_{0}$. It follows from the first and fourth equations of system (2) that for $t \geq t_{0}$,

$$
\begin{gathered}
S^{\prime}(t)>\mu \omega\left(1-v m_{I}^{*}\right)-\left(\mu+\beta m_{I}^{*}+\varepsilon \beta m_{I}^{*}\right) S+\varphi V, \\
V^{\prime}(t)>\mu(1-\omega)-\left[\mu+\varphi+\theta\left(\beta m_{I}^{*}+\varepsilon \beta m_{I}^{*}\right)\right] V .
\end{gathered}
$$

Consider the comparison impulsive system for $t \geq t_{0}$ :

$$
\begin{gathered}
u_{3}^{\prime}(t)=\mu \omega\left(1-v m_{I}^{*}\right)-\left(\mu+\beta m_{I}^{*}+\varepsilon \beta m_{I}^{*}\right) S+\varphi V, \\
u_{4}^{\prime}(t)=\mu(1-\omega)-\left(\mu+\varphi+\theta\left(\beta m_{I}^{*}+\varepsilon \beta m_{I}^{*}\right)\right) V, \\
t \neq n T, \quad n \in Z^{+}, \\
u_{3}\left(t^{+}\right)=(1-p) s_{1}(t), \\
u_{4}\left(t^{+}\right)=v_{1}(t)+p s_{1}(t), \\
t=n T, \quad n \in Z^{+} .
\end{gathered}
$$

According to Lemma 1, (35) have unique periodic solution

$$
\begin{aligned}
\widetilde{u}_{3}= & \frac{\mu \omega\left(1-v m_{I}^{*}\right)+\varphi}{\varphi+\mu+\beta m_{I}^{*}+\varepsilon \beta m_{I}^{*}} \\
& -\left(u_{4}^{*}-\frac{\mu(1-\omega)}{(\mu+\varphi) \theta\left(\beta m_{I}^{*}+\varepsilon \beta m_{I}^{*}\right)}\right) \\
& \times e^{-\left[\theta\left(\beta m_{I}^{*}+\varepsilon \beta m_{I}^{*}\right)+\mu+\varphi\right](t-n T)}, \\
\widetilde{u}_{4}= & \frac{\mu(1-\omega)}{(\mu+\varphi) \theta\left(\beta m_{I}^{*}+\varepsilon \beta m_{I}^{*}\right)} \\
& +\left(u_{4}^{*}-\frac{\mu(1-\omega)}{(\mu+\varphi) \theta\left(\beta m_{I}^{*}+\varepsilon \beta m_{I}^{*}\right)}\right) \\
& \times e^{-\left[\theta\left(\beta m_{I}^{*}+\varepsilon \beta m_{I}^{*}\right)+\mu+\varphi\right](t-n T)},
\end{aligned}
$$

where

$$
\begin{aligned}
u_{3}^{*}= & \left((1-p)\left(\mu \omega\left(1-v m_{I}^{*}\right)+\varphi\right)\right. \\
& \left.\times\left(1-e^{\left(\theta\left(\beta m_{I}^{*}+\varepsilon \beta m_{I}^{*}\right)+\mu+\varphi\right) T}\right)\right) \\
\times & \left(\left(\varphi+\mu+\beta m_{I}^{*}+\varepsilon \beta m_{I}^{*}\right)\right. \\
& \left.\times\left[1-(1-p) e^{-\left(\theta\left(\beta m_{I}^{*}+\varepsilon \beta m_{I}^{*}\right)+\mu+\varphi\right) T}\right]\right)^{-1}, \\
u_{4}^{*}=( & p\left(\varphi+\mu+\beta m_{I}^{*}+\varepsilon \beta m_{I}^{*}\right) \\
& \left.+\mu(1-p)(1-\omega)\left[1-e^{-\left(\theta\left(\beta m_{I}^{*}+\varepsilon \beta m_{I}^{*}\right)+\mu+\varphi\right) T}\right]\right) \\
\times & \left(\left(\varphi+\mu+\beta m_{I}^{*}+\varepsilon \beta m_{I}^{*}\right)\right. \\
& \left.\times\left[1-(1-p) e^{-\left(\theta\left(\beta m_{I}^{*}+\varepsilon \beta m_{I}^{*}\right)+\mu+\varphi\right) T}\right]\right)^{-1} .
\end{aligned}
$$

So there exists $T_{1}>t_{0}$ such that

$$
\begin{gathered}
S(t)>\widetilde{u}_{3}(t)-\bar{\varepsilon}=\eta_{1}, \\
V(t)>\widetilde{u}_{4}(t)-\bar{\varepsilon}=\eta_{2},
\end{gathered}
$$

for all $t>T_{1}$.

We denote

$$
\begin{aligned}
\Pi(t)= & I_{1}(t)+I_{2}(t) \\
& +\beta e^{-\mu \tau} \int_{t-\tau}^{t} I_{1}(\xi)[S(\xi)+\theta V(\xi)] d \xi \\
& +\beta \varepsilon e^{-\mu \tau} \int_{t-\tau}^{t} I_{2}(\xi)[S(\xi)+\theta V(\xi)] d \xi .
\end{aligned}
$$

From (2), we have

$$
\begin{aligned}
\Pi^{\prime}(t)= & {\left[\beta e^{-\mu \tau}(S+\theta V)-\left(\mu+(1-q) \gamma_{1}\right)\right] I_{1}(t) } \\
& +\left[\varepsilon \beta e^{-\mu \tau}(S+\theta V)+\mu v \omega-\left(\mu+\mu_{1}+\gamma_{2}\right)\right] I_{2}(t) \\
\geq & {\left[\beta e^{-\mu \tau}\left(\eta_{1}+\theta \eta_{2}\right)-\left(\mu+(1-q) \gamma_{1}\right)\right] I_{1}(t) } \\
& +\left[\varepsilon \beta e^{-\mu \tau}\left(\eta_{1}+\theta \eta_{2}\right)+\mu v \omega-\left(\mu+\mu_{1}+\gamma_{2}\right)\right] I_{2}(t)
\end{aligned}
$$

for $t>T_{1}$.

From (33), we obtain $\Pi^{\prime}(t)>0$, for $t>T_{1}$, which implies that $\Pi(t) \rightarrow \infty, t \rightarrow \infty$. This is contrary to the fact that $\Pi(t)$ is bounded. Hence, there exists a $t_{1}>0$ such that $I_{1}\left(t_{1}\right)+$ $I_{2}\left(t_{1}\right) \geq m_{I}^{*}$.

Next we prove that there exists a $m_{I}$ such that any positive solution of (2) satisfies $\lim _{t \rightarrow \infty} \inf I_{1}(t)+I_{2}(t)>m_{I}$.

Define

$$
m_{I}=\min \left\{\frac{m_{I}^{*}}{2}, q_{1}\right\}, \quad q_{1}=m_{I}^{*} e^{-\Lambda \tau},
$$

where $\Lambda=\max \left\{\mu+(1-q) \gamma_{1},(1-\omega v) \mu+\mu_{1}+\gamma_{2}\right\}$.

First, if $I_{1}(t)+I_{2}(t)>m_{I}^{*}$ for all $t>t_{1}$, then our aim is obtained.

Second $I_{1}(t)+I_{2}(t)$ oscillates about $m_{I}^{*}$ for all large $t$; setting $t^{*}=\inf _{t>t_{1}} I_{1}(t)+I_{2}(t) \leq m_{I}^{*}$, there are two possible cases for $t^{*}$.

We hope to show that $I_{1}(t)+I_{2}(t) \geq m_{I}$ for all large $t$. The conclusion is evident in the first case. For the second case, let $t^{*}>0$ and $\rho>0$ satisfy $I_{1}\left(t^{*}\right)+I_{2}\left(t^{*}\right)=I_{1}\left(t^{*}+\rho\right)+I_{2}\left(t^{*}+\rho\right)=$ $m_{I}^{*}$, and $I_{1}(t)+I_{2}(t)<m_{I}^{*}, S(t)>\eta$ for $t^{*}<t<t^{*}+\rho$. Therefore, it is certain that there exists a $g(0<g<\tau)$ such that

$$
I_{1}(t)+I_{2}(t) \geq \frac{m_{I}^{*}}{2} \quad \text { for } t^{*}<t<t^{*}+g .
$$

In this case, we will discuss three possible cases in terms of the sizes of $g, \rho$, and $\tau$.

Case I. If $\rho \leq g<\tau$, then $I_{1}(t)+I_{2}(t) \geq m_{I}^{*} / 2$ for $t^{*}<t<$ $t^{*}+\rho$.

Case II. If $g \leq \rho \leq \tau$, then from equations of system (2), we can deduce $I_{1}^{\prime}(t)+I_{2}^{\prime}(t)>-\Lambda I(t)$, where $\Lambda=\max \{\mu+(1-q)$ 


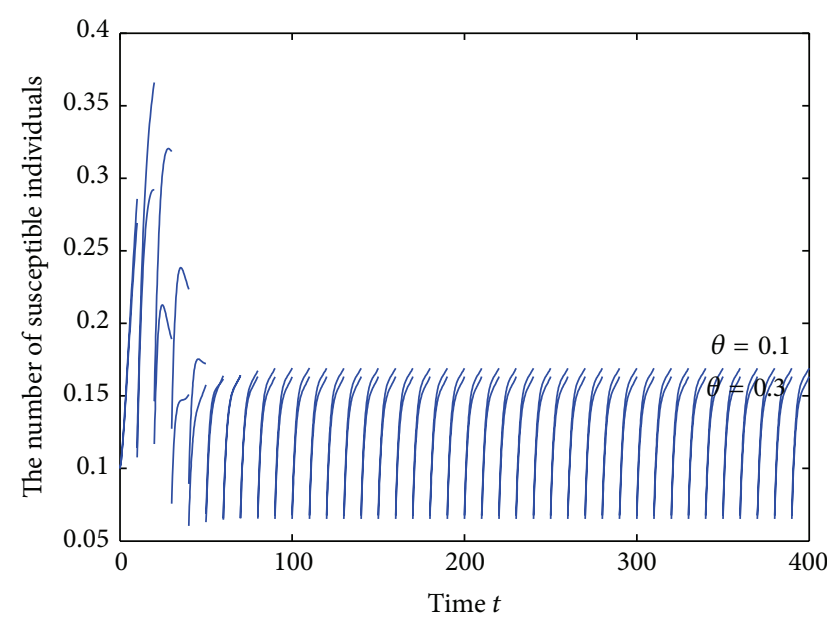

(a)

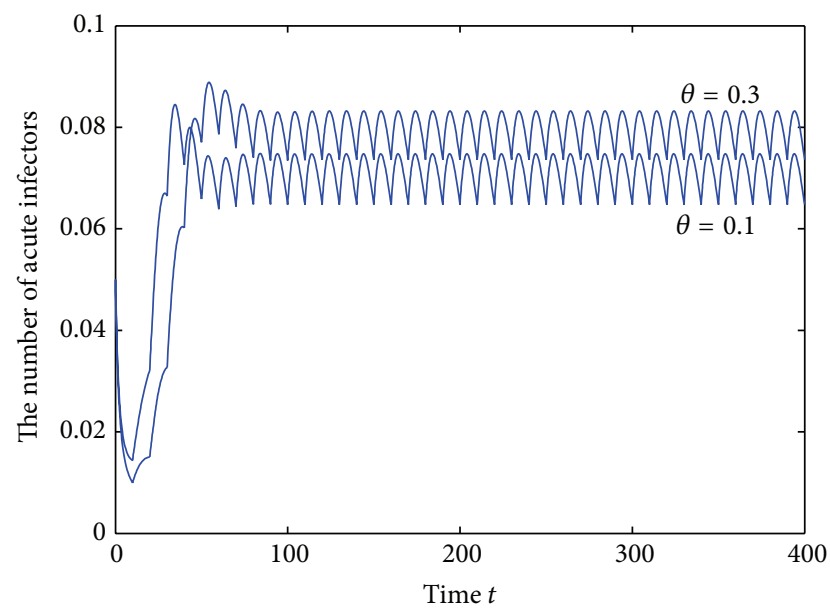

(c)

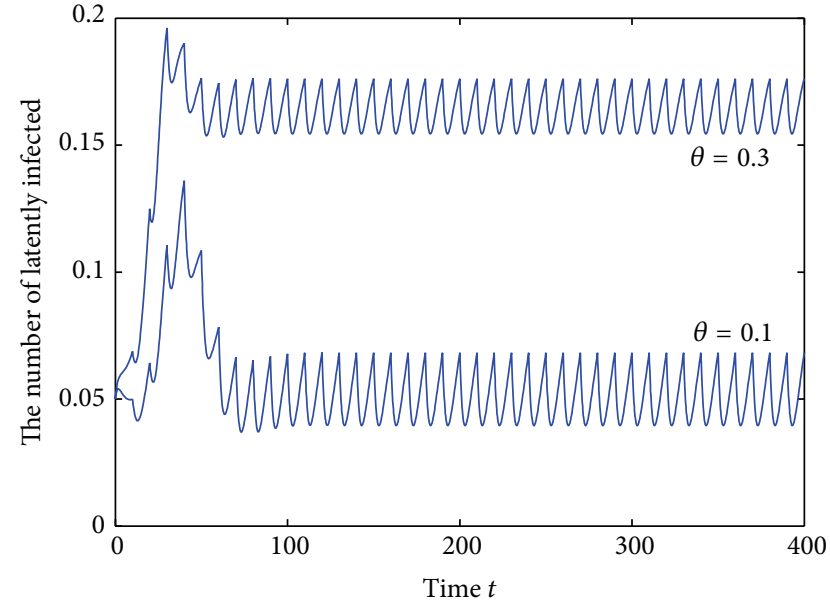

(b)

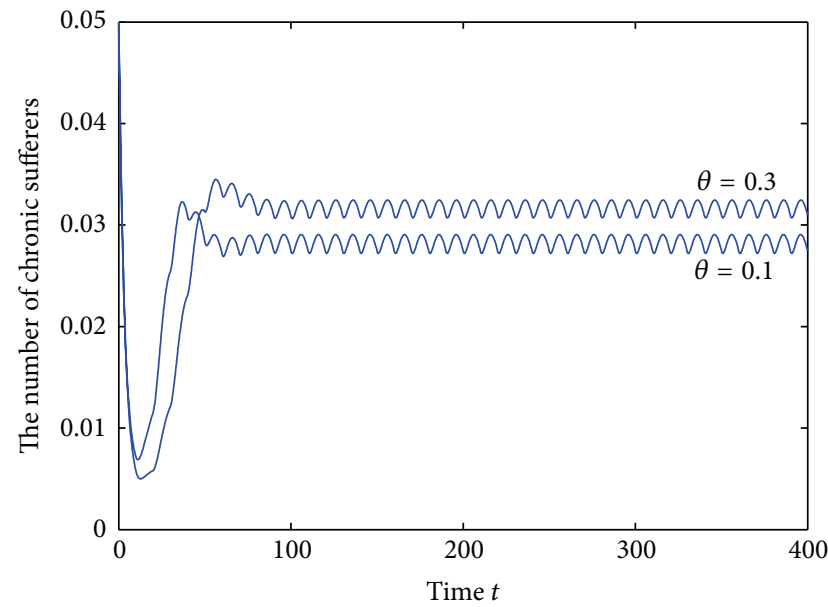

(d)

Figure 2: The effect of vaccine efficacy on the number of (a) susceptible individuals, (b) latently infected, (c) acute infectors, and (d) chronic sufferers. The parameters are $\mu=0.1 ; v=0.1 ; \varphi=0.1 ; \beta=3 ; \varepsilon=0.016 ; \gamma_{2}=0.025 ; \mu_{1}=0.28 ; \tau=10 ; \omega=0.2 ; q=0.4 ; \gamma_{1}=0.4 ; \theta=0.3$; $p=0.6$; the initial values are $S(0)=0.1 ; L(0)=0.05 ; I_{1}(0)=0.05 ; I_{2}(0)=0.05 ; V(0)=0.1 ; R(0)=0.1$.

$\left.\delta_{1},(1-\omega v) \mu+\mu_{1}+\gamma_{2}\right\}$ for $t \in\left[t^{*}, t^{*}+\tau\right]$ and $I_{1}\left(t^{*}\right)+I_{2}\left(t^{*}\right)=$ $m_{I}^{*}$; it is obvious that $I_{1}(t)+I_{2}(t) \geq q_{1}$ for $t^{*}<t<t^{*}+g$.

Case III. If $g \leq T \leq \rho$, we will consider the following two cases, respectively.

Case IIIa. For $t^{*}<t<t^{*}+\tau$, it is easy to obtain $I_{1}(t)+I_{2}(t)>$ $q_{1}$.

Case IIIb. For $t^{*}+\tau<t<t^{*}+\rho$, it is easy to obtain $I_{1}(t)+I_{2}(t)>q_{1}$. Then, proceeding exactly as the proof for the above claim, we see that $I_{1}(t)+I_{2}(t) \geq m_{I}$ for $t^{*}+\tau<t<$ $t^{*}+\rho$. Since this kind of interval $\left[t^{*}, t^{*}+\rho\right]$ is chosen in an arbitrary way (we only need $t^{*}$ to be large), we conclude that $I_{1}(t)+I_{2}(t) \geq m_{I}$ for all large $t$ in the second case. In view of our above discussions, the choices of $m_{I}$ are independent of the positive solution, and we have proved that any positive solution of (2) satisfies $I_{1}(t)+I_{2}(t) \geq m_{I}$ for all large $t$. The proof is completed.

\section{Numerical Simulations}

In this section, we present some numerical simulations to demonstrate the transmission dynamic of HBV. According to the natural history of $\mathrm{HBV}$ transmission and prior research $[10,12]$, we set some parameter values of the model: $\mu=0.1$; $v=0.1 ; \varphi=0.1 ; \beta=3 ; \varepsilon=0.016 ; \gamma_{2}=0.025 ; \mu_{1}=0.28$; $\tau=10 ; \omega=0.2 ; q=0.4 ; \gamma_{1}=0.4 ; \theta=0.3 ; p=0.6$. And we choose $\theta=0.1,0.3$, respectively; the number of susceptible individuals, latently infected, acute infectors and chronic sufferers are seen in Figure 2. It is easy to see that the lower of $\theta$ (the higher of vaccine efficacy) the fewer of the number of infected individuals. So, knowing the efficacy of $\mathrm{HBV}$ vaccine is necessary for the extinct of disease.

In order to find better control strategies for HBV infection, we would like to see what parameters can affect the change of the acute infectors number. From Figure 3(a) we can see that the increase of $p$ and decrease of $\theta$ will make the number of acute infectors lower. If $\theta$ is higher, that is to say, 


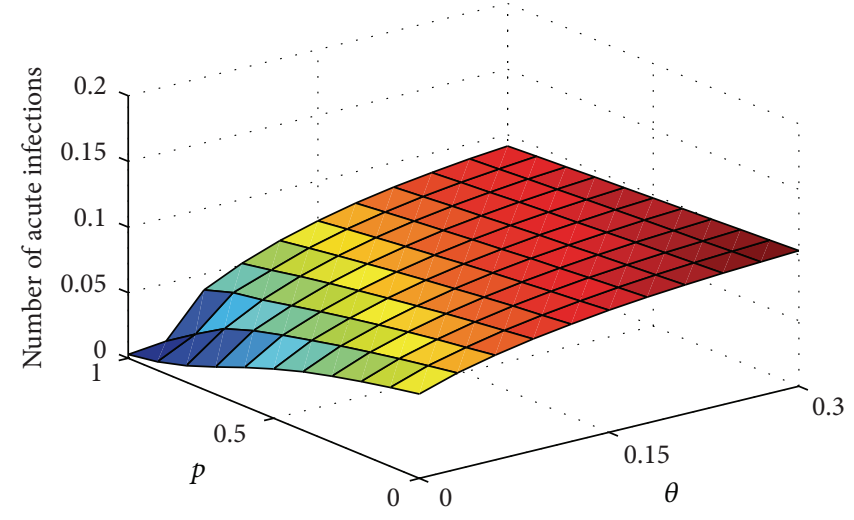

(a)

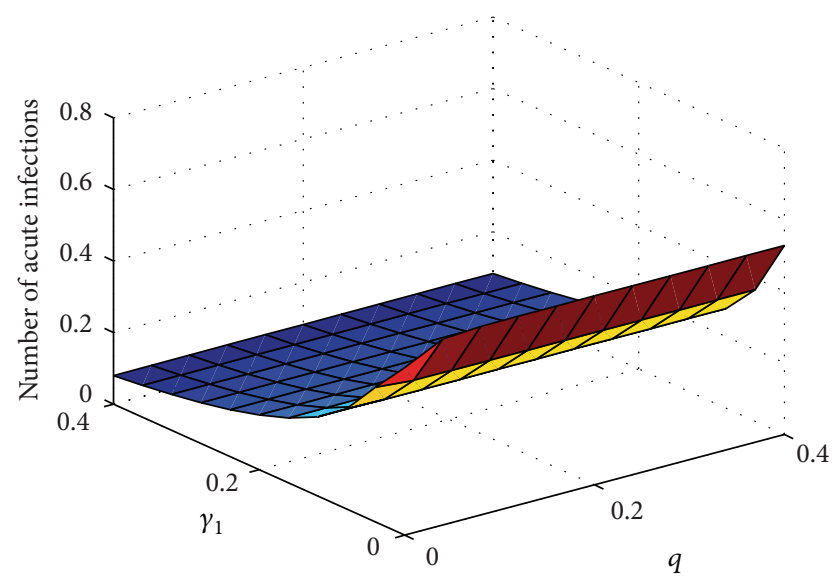

(c)

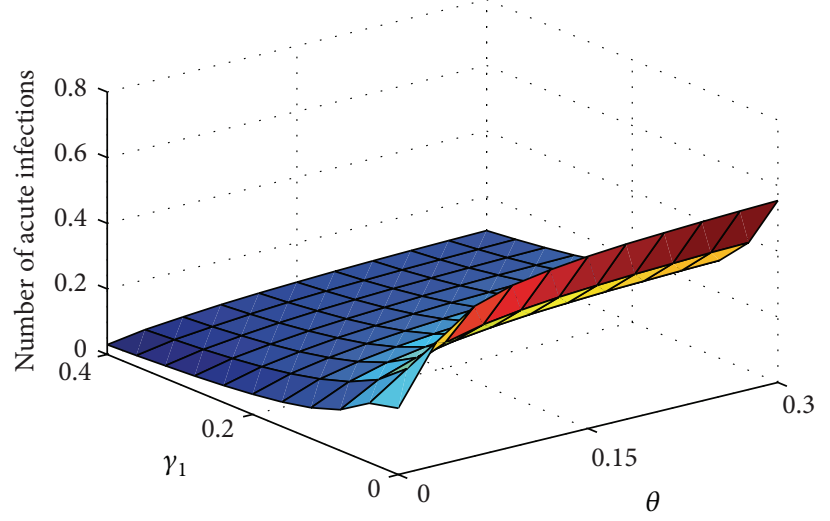

(b)

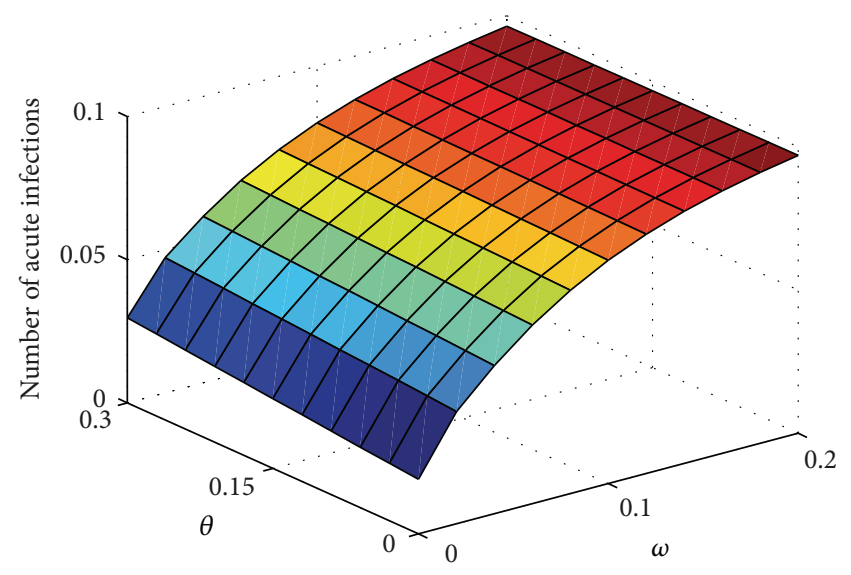

(d)

FIGURE 3: The graphs of the number of acute infectors with some parameters: (a) $\mu=0.1 ; v=0.1 ; \varphi=0.1 ; \beta=12 ; \varepsilon=0.16 ; \gamma_{2}=0.025$; $\mu_{1}=0.028 ; \tau=5 ; \gamma_{1}=0.4 ; q=0.4 ; \omega=0.2$, surf $p \in[0,1], \theta \in[0,0.3]$. (b) $\mu=0.1 ; v=0.1 ; \varphi=0.1 ; \beta=12 ; \varepsilon=0.16 ; \gamma_{2}=0.025 ; \mu_{1}=0.028$; $\tau=5 ; \omega=0.2 ; q=0.4 ; p=0.6, \operatorname{surf} \theta \in[0,0.3], \gamma_{1} \in[0,0.4]$. (c) $\mu=0.1 ; v=0.1 ; \varphi=0.1 ; \beta=12 ; \varepsilon=0.16 ; \gamma_{2}=0.025 ; \mu_{1}=0.028 ; \tau=5$; $\omega=0.2 ; \theta=0.2 ; p=0.8$, surf $\gamma_{1} \in[0,0.4], q \in[0,0.4]$. (d) $\mu=0.1 ; v=0.1 ; \varphi=0.1 ; \beta=12 ; \varepsilon=0.16 ; \gamma_{2}=0.025 ; \mu_{1}=0.028 ; \tau=5 ; \gamma_{1}=0.4$; $q=0.4 ; p=0.6, \operatorname{surf} \theta \in[0,0.3], \omega \in[0,0.2]$.

the efficacy of vaccination is lower, higher vaccination cannot keep the disease extinct. So, to control the disease spread, knowing the vaccine efficacy is important.

In Figure 3(b) we plot the number of acute infectors which change with parameters $\theta$ and $\gamma_{1}$. We can see that with the increase of $\gamma_{1}$ the number of acute infectors decreases even if the efficacy of vaccination is lower. That is to say, take measures to cure the acute infectors in time is a necessary method to decrease the number of acute infectors. So, doing check regularly and treatment at early time are necessary. In Figure 3(c) we find not only the increase of $\gamma_{1}$ but also the parameter $q$ has effect on the number of acute infections; the higher the acute individuals treatment rate, the lower the number of acute infections.

In Figure 3(d), we detect that the decrease of $\theta$ and $\omega$ will make the number of infected individuals lower. So successful immunization of both newborns and susceptible individuals is an efficient intervention strategy. The optimal control strategy will be a combination of improving the vaccine efficacy, increasing the immunization of newborns and susceptible individuals, and increasing the treatment to the acute infected individuals.

\section{Conclusion}

Hepatitis B virus is highly prevalent in many countries of the world; we promote a new epidemic model based on the spread characters of the HBV, and consider the fact that the HepB vaccine is incomplete immunization in the vaccination process. Our model is more approach to the realistic problem and different from $[13,14]$. Moreover, the methods in disposing the model are different from the existing results because more factors are considered. We find when $\mathfrak{R}_{1}<1$ and $\Re_{2}<1$; the disease-free periodic solution is globally attractive; if $\Re_{1}>1$ and $\Re_{2}>1$, the disease is permanent by using the comparison theorem of impulsive differential equation. By some simulation experiments, Figures 2 and 3 show some effects of parameters on the number of acute individuals and provides an optimal control strategy for the HBV transmission. 


\section{Conflict of Interests}

The authors declare that there is no conflict of interests regarding the publication of this paper.

\section{Acknowledgment}

The work is supported by the National Natural Science Foundation of China (no. 1124319).

\section{References}

[1] World Health Organization, Hepatitis B, WHO/CDS/CSR/ LYO/2002.2: Hepatitis B, 2002, http://www.who.int/csr/disease/ hepatitis/whocdscsrlyo20022/en/.

[2] New Zealand Ministry of Health, Immunisation Handbook 2006, New Zealand Ministry of Health, Wellington, New Zealand, 2006.

[3] J.-D. Jia, "Hepatitis B in China: From guideline to practice," Virologica Sinica, vol. 23, no. 2, pp. 152-155, 2008.

[4] S. J. Liu and Q. Zhu, HBV Disabusing and Answering Doubt, Chinese Medicine and Scientific Technology, Beijing, China, 2003.

[5] B. Ezquieta, E. Cueva, A. Oliver, and R. Gracia, "SHOX intrag -enic microsatellite analysis in patients with short stature," Journal of Pediatric Endocrinology and Metabolism, vol. 15, no. 2, pp. 139-148, 2002.

[6] F.-M. Lu and H. Zhuang, "Management of hepatitis B in China," Chinese Medical Journal, vol. 122, no. 1, pp. 3-4, 2009.

[7] World Health Organisation, "Hepatitis B vaccines. Position paper," Weekly Epidemiol Record, vol. 40, pp. 405-420, 2009.

[8] New Zealand Ministry of Health, Immunisation Handbook 2002, New Zealand Ministry of Health, Wellington, New Zealand, 2002.

[9] R. M. Anderson and R. M. May, Disease of Humans: Dynamics and Control, Oxford University Press, Oxford, UK, 1991.

[10] S. Thornley, C. Bullen, and M. Roberts, "Hepatitis B in a high prevalence New Zealand population: a mathematical model applied to infection control policy," Journal of Theoretical Biology, vol. 254, no. 3, pp. 599-603, 2008.

[11] G. F. Medley, N. A. Lindop, W. J. Edmunds, and D. J. Nokes, "Hepatitis-B virus endemicity: Heterogeneity, catastrophic dynamics and control," Nature Medicine, vol. 7, no. 5, pp. 619-624, 2001.

[12] L. Zou, W. Zhang, and S. Ruan, "Modeling the transmission dynamics and control of hepatitis B virus in China," Journal of Theoretical Biology, vol. 262, no. 2, pp. 330-338, 2010.

[13] J. Pang, J.-a. Cui, and X. Zhou, "Dynamical behavior of a hepatitis B virus transmission model with vaccination," Journal of Theoretical Biology, vol. 265, no. 4, pp. 572-578, 2010.

[14] S. Zhang and Y. Zhou, "The analysis and application of an HBV model," Applied Mathematical Modelling, vol. 36, no. 3, pp.13021312, 2012.

[15] L. Zou, S. Ruan, and W. Zhang, "An age-structured model for the transmission dynamics of hepatitis B," SIAM Journal on Applied Mathematics, vol. 70, no. 8, pp. 3121-3139, 2010.

[16] K. Wang, W. Wang, and S. Song, "Dynamics of an HBV model with diffusion and delay," Journal of Theoretical Biology, vol. 253, no. 1, pp. 36-44, 2008.

[17] R. Xu and Z. Ma, "An HBV model with diffusion and time delay," Journal of Theoretical Biology, vol. 257, no. 3, pp. 499-509, 2009.
[18] M. A. Khan, S. Islam, M. Arif, and Z. Ul Haq, "Transmission model of hepatitis b virus with the migration effect," BioMed Research International, vol. 2013, Article ID 150681, 10 pages, 2013.

[19] A. Gabbuti, L. Romanò, P. Blanc et al., "Long-term immunogenicity of hepatitis B vaccination in a cohort of Italian healthy adolescents," Vaccine, vol. 25, no. 16, pp. 3129-3132, 2007.

[20] X. Liu, Y. Takeuchi, and S. Iwami, "SVIR epidemic models with vaccination strategies," Journal of Theoretical Biology, vol. 253, no. 1, pp. 1-11, 2008.

[21] Y. Xiao and S. Tang, "Dynamics of infection with nonlinear incidence in a simple vaccination model," Nonlinear Analysis: Real World Applications, vol. 11, no. 5, pp. 4154-4163, 2010.

[22] J. Li and Y. Yang, "SIR-SVS epidemic models with continuous and impulsive vaccination strategies," Journal of Theoretical Biology, vol. 280, no. 1, pp. 108-116, 2011.

[23] X. Song, Y. Jiang, and H. Wei, "Analysis of a saturation incidence SVEIRS epidemic model with pulse and two time delays," Applied Mathematics and Computation, vol. 214, no. 2, pp. 381390, 2009.

[24] Y. Jiang, H. Wei, X. Song, L. Mei, G. Su, and S. Qiu, "Global attractivity and permanence of a delayed SVEIR epidemic model with pulse vaccination and saturation incidence," Applied Mathematics and Computation, vol.213, no. 2, pp. 312-321, 2009.

[25] M. Qiao, H. Qi, and Y. Chen, "Qualitative analysis of hepatitis $B$ virus infection model with impulsive vaccination and time delay," Acta Mathematica Scientia B, vol. 31, no. 3, pp. 1020-1034, 2011.

[26] M. Qiao, A. Liu, and U. Foryś, "Qualitative analysis of the SICR epidemic model with impulsive vaccinations," Mathematical Methods in the Applied Sciences, vol. 36, no. 6, pp. 695-706, 2013.

[27] V. Lakshmikantham, D. D. Baĭnov, and P. S. Simeonov, Theory of Impulsive Differential Equations, vol. 6 of Series in Modern Applied Mathematics, World Scientific, Singapore, 1989.

[28] Y. Kuang, Delay Differential Equations with Applications in Population Dynamics, vol. 191 of Mathematics in Science and Engineering, Academic Press, New York, NY, USA, 1993. 


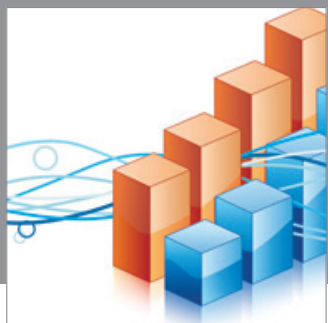

Advances in

Operations Research

mansans

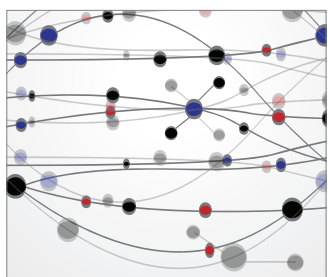

The Scientific World Journal
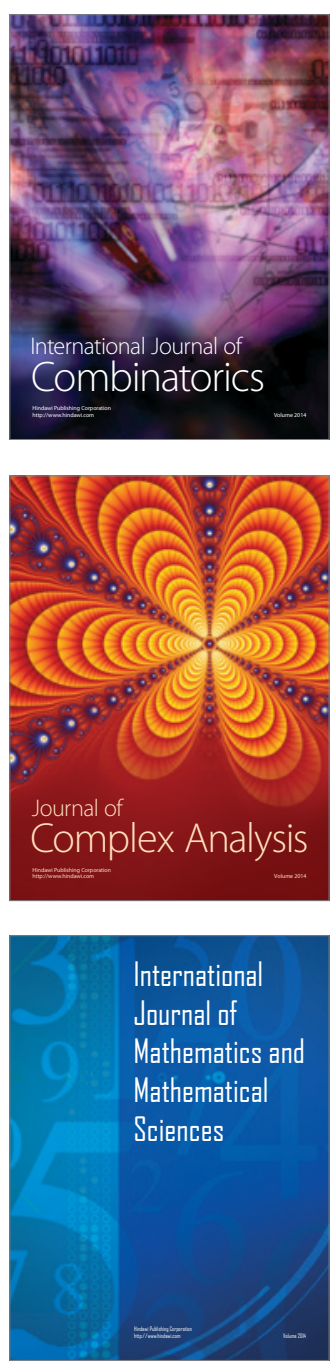
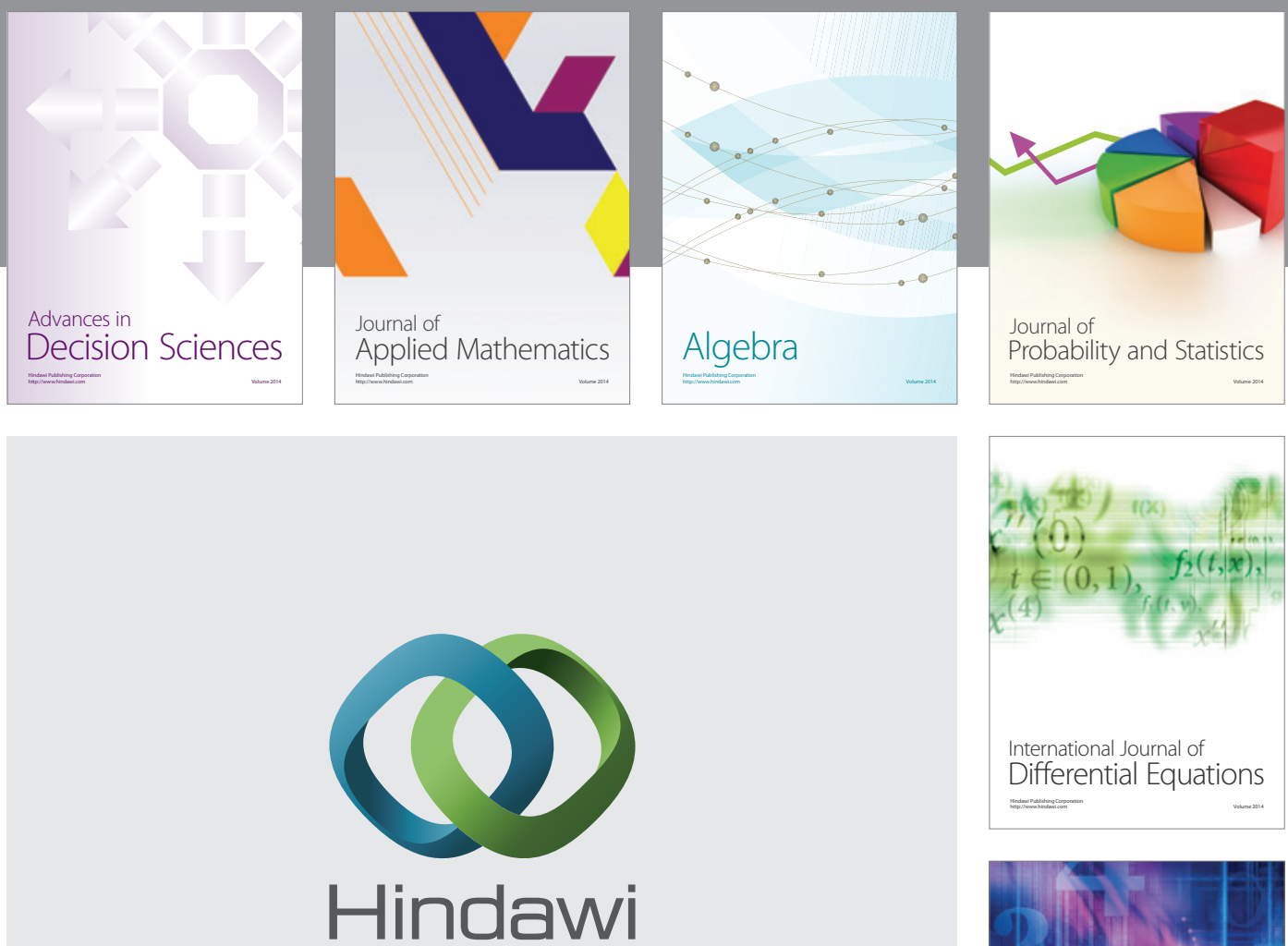

Submit your manuscripts at http://www.hindawi.com
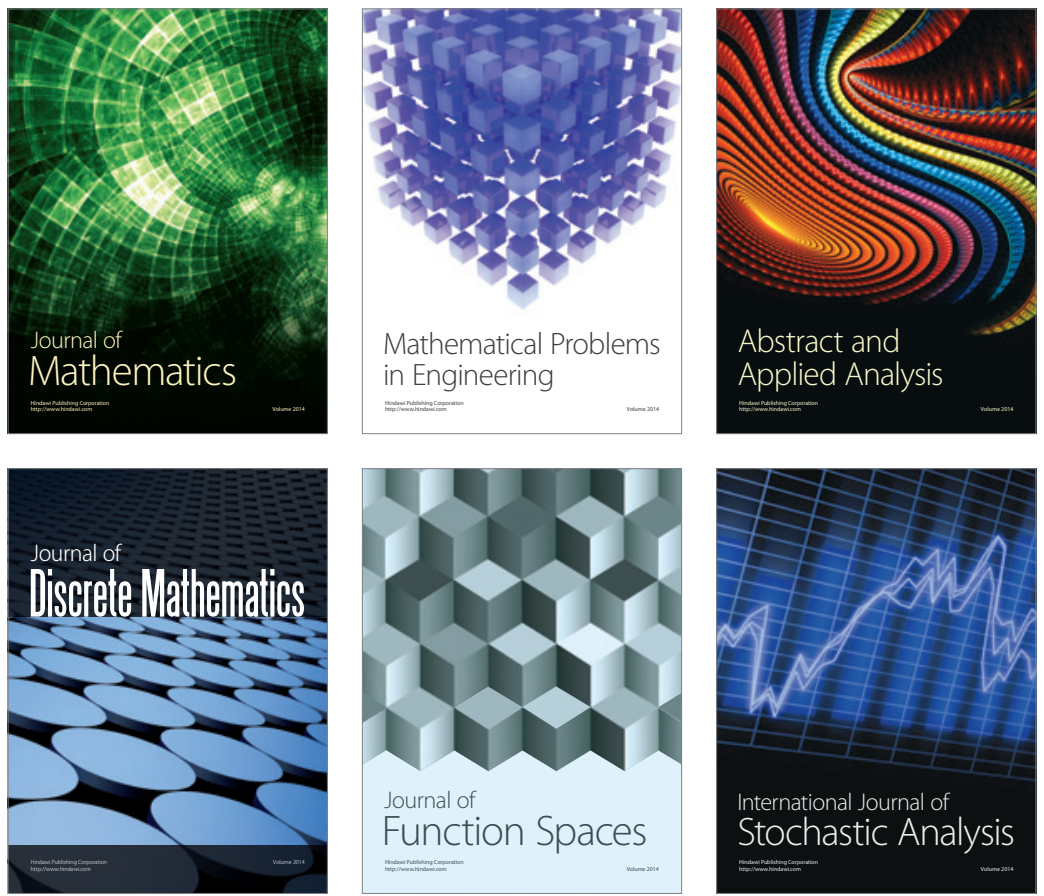

Journal of

Function Spaces

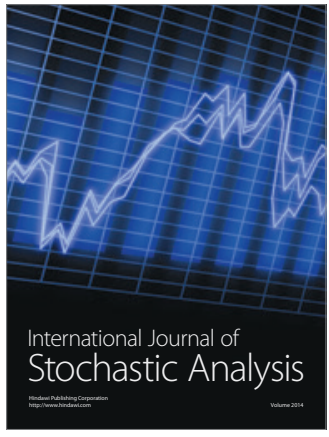

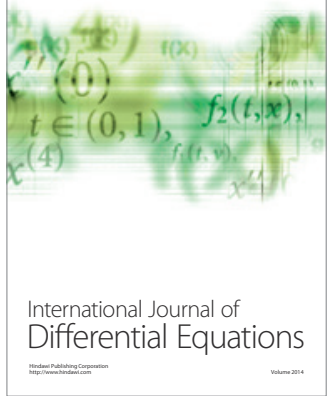
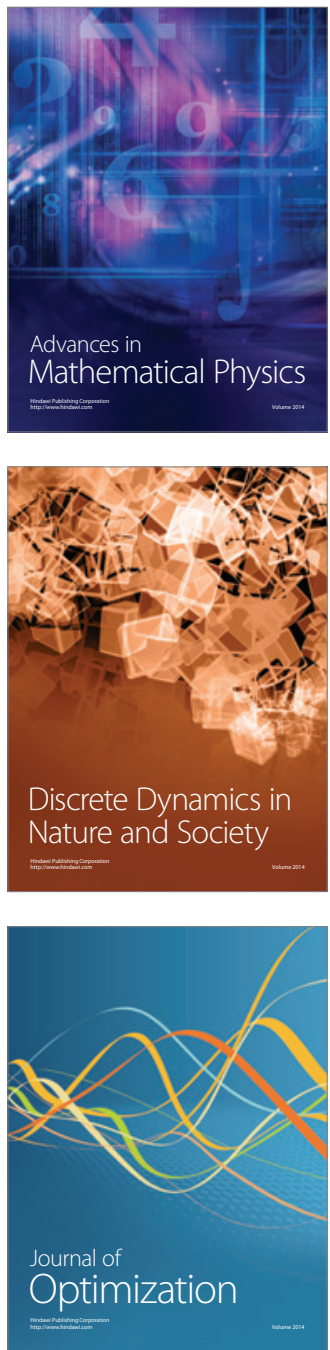